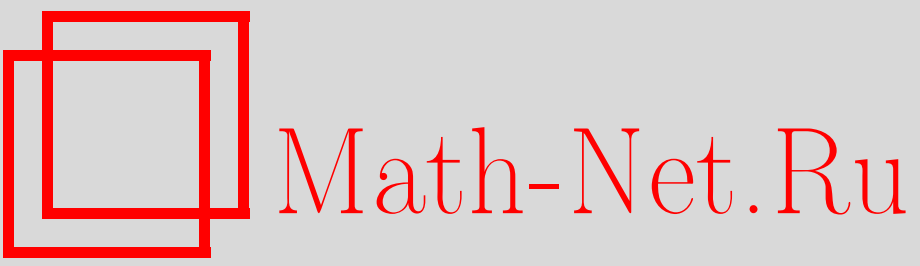

Ф. К. Клебанер, Р. Ш. Липцер, Диффузионная модель разорения. Асимптотический анализ, Теория вероятн. и ее примен., 2010, том 55, выпуск 2, 350-357

DOI: https://doi.org/10.4213/tvp4205

Использование Общероссийского математического портала Math-Net.Ru подразумевает, что вы прочитали и согласны с пользовательским соглашением

http://www.mathnet.ru/rus/agreement

Параметры загрузки:

IP : 34.239 .49 .27

26 апреля 2023 г., 16:51:39 
5. Райгородский A.M. Линейно-алгебраический метод в комбинаторике. М.: МЦНМО, 2007, 136 с.

6. Bollobás B. Random Graphs. Cambridge: Cambridge Univ. Press, 2001, 498 p.

7. Колиин В. Ф. Случайные графы. М.: Физматлит, 2004, 255 с.

8. Верещагин Н. К., Шень А. Языки и исчисления. М.: МЦНМО, 2000, 286 с.

9. Schwentick T. On winning Ehrenfeucht games and monadic NP. - Ann. Pure Appl. Logic, 1996, v. 79, № 1, p. 61-92.

10. Успенский В. А., Верещагин Н. К., Плиско В.Е. Вводный курс математической логики. М.: Физматлит, 2007, 125 с.

11. Ehrenfeucht $A$. An application of games to the completeness problem for formalized theories. - Fund. Math., 1960, v. 49, p. 129-141.

12. Алон H., Спенсер Дж. Вероятностный метод. М.: БИНОМ. Лаб. знаний, 2007, $320 \mathrm{c}$

Поступила в редакцию 5.VIII.2009

(C) 2010 г.

\author{
КЛЕБАНЕР Ф.*, ЛИПЦЕР Р.Ш.**
}

\title{
ДИФФУЗИОННАЯ МОДЕЛЬ РАЗОРЕНИЯ. АСИМПТОТИЧЕСКИЙ АНАЛИЗ
}

Изучается вероятность поглощения $\mathbf{P}\left(\tau_{0} \leqslant T\right)$ на интервале $[0, T]$, где $\tau_{0}=\inf \left\{t: X_{t}=0\right\}$ и $X_{t}$ - неотрицательный диффузионный процесс относительно броуновского движения $B_{t}$ :

$$
d X_{t}=\mu X_{t} d t+\sigma X_{t}^{\gamma} d B_{t}, \quad X_{0}=K>0 .
$$

Диффузионный коэффициент $\sigma x^{\gamma}, \gamma \in[1 / 2,1)$, не удовлетворяет условию Липшица и тем самым обеспечивает выполнение неравенства $\mathbf{P}\left(\tau_{0} \leqslant\right.$ $T)>0$. Наш основной результат:

$$
\lim _{K \rightarrow \infty} \frac{1}{K^{2(1-\gamma)}} \ln \mathbf{P}\left(\tau_{0} \leqslant T\right)=-\frac{1}{2 \mathbf{E} M_{T}^{2}},
$$

где $M_{t}=\int_{0}^{t} \sigma(1-\gamma) e^{-(1-\gamma) \mu s} d B_{s}$. Кроме того, мы даем описание наиболее вероятной траектории поглощения нормированного процесса $X_{t} / K$ при $K \rightarrow \infty$.

Ключевые слова и фразы: диффузионный процесс, вероятность поглощения, принцип больших уклонений.

1. Введение. В данной статье мы рассматриваем известную модель:

$$
\text { Constant Elasticity of Variance Model, }
$$

введенную в [1] и нашедшую применение в моделировании цены опционов (см., например, [2] и [9]).

Эта модель задается уравнением Ито со стандартным броуновским движением $B_{t}$ и положительным начальным условием $X_{0}=K>0$ :

$$
d X_{t}=\mu X_{t} d t+\sigma X_{t}^{\gamma} d B_{t},
$$

где $\mu, \sigma$ - произвольные отличные от нуля константы и $\gamma \in[1 / 2,1)$. В частном случае $\gamma=1 / 2$ эта модель известна как модель Кокса-Ингерсола-Росса. Несмотря на то что

* School of Mathematical Sciences, Building 28M, Monash University, Clayton Campus, Victoria 3800, Australia; e-mail: fima.klebaner@sci.monash.edu.au

** Department of Electrical Engineering Systems, Tel Aviv University, 69978 Tel Aviv, Israel; e-mail: liptser@eng.tau.ac.il; rliptser@gmail.com 
коэффициент диффузии $\sigma x^{\gamma}$ удовлетворяет только условию Гёльдера, уравнение (1.1) имеет единственное сильное неотрицательное решение (существование доказано Делбаэном и Ширакавой [2], единственность установлена Ямадой и Ватанабэ - см., например, $[10$, с. 265$]$ или $[6$, с. 17 и теорема 13.1]). В отличие от модели с $\gamma=1$ (модель Блэка-Шоулса), где процесс $X_{t}$ остается положительным при любом $t>0$, в рассматриваемой модели $X_{t}$ может обращаться в нуль в момент времени $\tau_{0}=\inf \left\{t: X_{t}=0\right\}$ с $\mathbf{P}\left(\tau_{0}<\infty\right)>0$, который можно трактовать как момент разорения.

Центральную роль в предлагаемом асимптотическом анализе $(K \rightarrow \infty)$ играет нормированный процесс $x_{t}^{K}=X_{t} / K$, определяемый уравнением Ито

$$
d x_{t}^{K}=\mu x_{t}^{K} d t+\frac{\sigma}{K^{1-\gamma}}\left(x_{t}^{K}\right)^{\gamma} d B_{t}
$$

с начальным условием $x_{0}^{K}=1$ и малым диффузионным параметром $\sigma x^{\gamma} / K^{1-\gamma}$, для которого момент разорения $\tau_{0}$ сохраняется.

При $K \rightarrow \infty$ мы привлекаем результаты из теории больших уклонений для двух семейств:

$$
\left\{\left(x_{t}^{K}\right)_{t \in[0, T]}\right\}_{K \rightarrow \infty} \quad \text { и }\left\{\left(\frac{1}{K^{1-\gamma}} M_{t}\right)_{t \in[0, T]}\right\}_{K \rightarrow \infty},
$$

где

$$
M_{t}=\int_{0}^{t} \sigma(1-\gamma) e^{-(1-\gamma) \mu s} d B_{s}
$$

Для второго семейства принцип больших уклонений (ПБУ) хорошо известен. Для первого семейства ПБУ Вентцеля-Фрейдлина [5] ожидаем, несмотря на то, что его диффузионный параметр вырождается в нуле и удовлетворяет лишь условию Гёльдера. В частности, для $\gamma=1 / 2$ ПБУ известен (см. [3]) с параметром $1 / K$ и функционалом действия Вентцеля-Фрейдлина с некоторой коррекцией: $J_{T}(u)=$ $1 / 2 \int_{0}^{T}\left(\left(\dot{u}_{t}-\mu u_{t}\right) / \sqrt{u_{t}}\right)^{2} I_{\left\{u_{t}>0\right\}} d t$ (по поводу $\gamma=1 / 2$ см. также [7] и [11]).

Мы показываем, что в случае $\gamma \in(1 / 2,1)$ ПБУ также имеет место с коэффициентом и функционалом действия, зависящими от $\gamma$. Комбинируя оба ПБУ, мы получаем следующий асимптотический результат: существует гладкая функция $u_{t}^{*}$ с $u_{0}^{*}=1$, поглощаемая в момент времени $T$, такая, что для любой другой гладкой неотрицательной функции $u_{t}$ с $u_{0}=1$, поглощаемой на интервале $[0, T]$, выполнено

$$
\begin{aligned}
\lim _{K \rightarrow \infty} & \frac{1}{K^{2(1-\gamma)}} \ln \mathbf{P}\left(\tau_{0} \leqslant T\right) \\
\quad & =\lim _{\delta \rightarrow 0} \lim _{K \rightarrow \infty} \frac{1}{K^{2(1-\gamma)}} \ln \mathbf{P}\left(\sup _{t \in[0, T]}\left|x_{t}^{K}-u_{t}^{*}\right| \leqslant \delta\right) \\
& \geqslant \lim _{\delta \rightarrow 0} \lim _{K \rightarrow \infty} \frac{1}{K^{2(1-\gamma)}} \ln \mathbf{P}\left(\sup _{t \in[0, T]}\left|x_{t}^{K}-u_{t}\right| \leqslant \delta\right) .
\end{aligned}
$$

Последнее неравенство позволяет нам рассматривать $u_{t}^{*}$ как наиболее правдоподобную траекторию поглощения нормированного процесса $x_{t}^{K}$.

Отметим, что вычисление вероятности $\mathbf{P}\left(\tau_{0} \leqslant T\right)$ в логарифмической шкале требует применения нестандартной техники.

Имеем $\left\{\tau_{0} \leqslant T\right\}=\left\{\left(x_{t}^{K}\right)_{t \in[0, T]} \in \mathbf{D}\right\}$, где

$$
\mathbf{D}=\left\{u \in \mathbf{C}_{[0, T]}: u_{0}=1 ; u_{t}=u_{\theta(u) \wedge t}, \text { где } \theta(u)=\inf \left\{t: u_{t}=0\right\} \leqslant T\right\} .
$$

Множество $\mathbf{D}$ замкнуто в равномерной метрике $\varrho$ пространства $\mathbf{C}_{[0, T]}$ непрерывных функций на отрезке $[0, T]$. Поэтому верхний предел

$$
\varlimsup_{K \rightarrow \infty} \frac{1}{K^{2(1-\gamma)}} \ln \mathbf{P}\left(\tau_{0} \leqslant T\right)
$$


вычисляется в соответствии с ПБУ. Однако внутренняя часть $\mathbf{D}^{\circ}$ множества $\mathbf{D}$ есть пустое множество, $\mathbf{D}^{\circ}=\varnothing$. Этот факт исключает применение ПБУ для вычисления нижней границы

$$
\varliminf_{K \rightarrow \infty} \frac{1}{K^{2(1-\gamma)}} \ln \mathbf{P}\left(\tau_{0} \leqslant T\right) .
$$

Этой проблемы можно избежать, используя включение

$$
\left\{\tau_{0} \leqslant T\right\} \supseteq\left\{\frac{1}{K^{1-\gamma}} M_{T}<-1\right\}
$$

$\left(M_{T}\right.$ определено в (1.3)) и удивительный результат

$$
\lim _{K \rightarrow \infty} \frac{1}{K^{2(1-\gamma)}} \ln \left\{\frac{1}{K^{1-\gamma}} M_{T}<-1\right\}=\varlimsup_{K \rightarrow \infty} \frac{1}{K^{2(1-\gamma)}} \ln \mathbf{P}\left(\tau_{0} \leqslant T\right),
$$

который вместе с очевидным неравенством

$$
\lim _{K \rightarrow \infty} \frac{1}{K^{2(1-\gamma)}} \ln \left\{\frac{1}{K^{1-\gamma}} M_{T}<-1\right\} \leqslant \varliminf_{K \rightarrow \infty} \frac{1}{K^{2(1-\gamma)}} \ln \mathbf{P}\left(\tau_{0} \leqslant T\right)
$$

позволяет установить требуемый результат для $\mathbf{P}\left(\tau_{0} \leqslant T\right)$ в логарифмической шкале.

Этот подход интересен сам по себе и может оказаться полезным для анализа ПБУ и в других моделях.

2. Асимптотика при $K \rightarrow \infty$ для семейства $\mathbf{P}\left(\tau_{0} \leqslant T\right)$ в логарифмической шкале. Случайный процесс $M_{t}$, определенный в (1.3), является гауссовским мартингалом с квадратической характеристикой $\langle M\rangle_{t}$, совпадающей с $\mathbf{E} M_{t}^{2}$ :

$$
\langle M\rangle_{t}=\int_{0}^{t} \sigma^{2}(1-\gamma)^{2} e^{-2(1-\gamma) \mu s} d s
$$

Теорема 2.1. Для любого $T>0$

$$
\lim _{K \rightarrow \infty} \frac{1}{K^{2(1-\gamma)}} \ln \mathbf{P}\left(\tau_{0} \leqslant T\right)=-\frac{1}{2 \mathbf{E} M_{T}^{2}} .
$$

Д о к а з а т е л ь с т в о. Для того чтобы пользоваться формулой Ито в окрестности $\tau_{0}$, зададим момент остановки $\tau_{\varepsilon}=\inf \left\{t \leqslant T: x_{t}^{K}=\varepsilon\right\}, \varepsilon>0$. Теперь, применяя формулу Ито к $\left(x_{t}^{K}\right)^{1-\gamma}, t \leqslant \tau_{\varepsilon} \wedge T$, получаем

$$
\begin{aligned}
\left(x_{t}^{K}\right)^{1-\gamma}= & +\int_{0}^{t}(1-\gamma) \mu\left(x_{s}^{K}\right)^{1-\gamma} d s+\int_{0}^{t}(1-\gamma) \sigma \frac{d B_{s}}{K^{1-\gamma}} \\
& -\frac{1}{2} \int_{0}^{t} \gamma(1-\gamma) \frac{\sigma^{2}}{K^{2(1-\gamma)}} \frac{1}{\left(x_{s}^{K}\right)^{1-\gamma}} d s
\end{aligned}
$$

и затем

$$
\begin{aligned}
& \left(x_{\tau_{\varepsilon} \wedge T}^{K}\right)_{\tau_{\varepsilon} \wedge T}^{1-\gamma} e^{-(1-\gamma) \mu\left(\tau_{\varepsilon} \wedge T\right)} \\
& +\int_{0}^{\tau_{\varepsilon} \wedge T} \frac{\sigma^{2}}{2 K^{2(1-\gamma)}} \gamma(1-\gamma) \frac{e^{(1-\gamma) \mu s}}{\left(x_{s}^{K}\right)^{1-\gamma}} d s=1+\frac{1}{K^{1-\gamma}} M_{\tau_{\varepsilon} \wedge T} .
\end{aligned}
$$

Поскольку $\lim _{\varepsilon \rightarrow 0} M_{\tau_{\varepsilon} \wedge T}=M_{\tau_{0} \wedge T}$ п.н., то по теореме о монотонной сходимости

$$
\begin{aligned}
\lim _{\varepsilon \rightarrow 0} & \int_{0}^{\tau_{\varepsilon} \wedge T} \frac{\sigma^{2}}{2 K^{2(1-\gamma)}} \gamma(1-\gamma) \frac{e^{(1-\gamma) \mu s}}{\left(x_{s}^{K}\right)^{1-\gamma}} d s \\
& =\int_{\left[0, \tau_{0} \wedge T\right)} \frac{\sigma^{2}}{2 K^{2(1-\gamma)}} \gamma(1-\gamma) \frac{e^{(1-\gamma) \mu s}}{\left(x_{s}^{K}\right)^{1-\gamma}} d s \quad \text { п.н.; }
\end{aligned}
$$


следовательно, переходя в обеих частях равенства $(2.3)$ к пределу по $\varepsilon \rightarrow 0$, получаем

$$
\begin{aligned}
0 \leqslant & \left(x_{\tau_{0} \wedge T}^{K}\right)^{1-\gamma} e^{-(1-\gamma) \mu\left(\tau_{0} \wedge T\right)} \\
& +\int_{\left[0, \tau_{0} \wedge T\right)} \frac{\sigma^{2}}{2 K^{2(1-\gamma)}} \gamma(1-\gamma) \frac{e^{(1-\gamma) \mu s}}{\left(x^{K}\right)_{s}^{1-\gamma}} d s=1+\frac{1}{K^{1-\gamma}} M_{\tau_{0} \wedge T} .
\end{aligned}
$$

Из (2.4) вытекает неравенство $1+M_{\tau_{0} \wedge T} / K^{1-\gamma} \geqslant 0$. Другими словами, $\omega \in\left\{\tau_{0}>T\right\}$ означает, что $1+M_{T}(\omega) / K^{1-\gamma} \geqslant 0$. Следовательно, имеет место включение $\left\{\tau_{0}>\right.$ T\} $\subset\left\{1+M_{T} / K^{1-\gamma} \geqslant 0\right\}$ и, значит,

$$
\left\{\tau_{0} \leqslant T\right\} \supseteq\left\{\frac{1}{K^{1-\gamma}} M_{T}+1<0\right\} .
$$

Хорошо известно, что семейство $\left\{M_{T} / K^{1-\gamma}\right\}_{K \rightarrow \infty}$ удовлетворяет ПБУ в метрическом пространстве $(\mathbf{R}, \rho)$ (здесь $\rho-$ евклидова метрика) с параметром $1 / K^{2(1-\gamma)}$ и функционалом действия $I(v)=v^{2} /\left(2 \mathbf{E} M_{T}^{2}\right)$. В соответствии с теорией больших уклонений,

$$
\varliminf_{K \rightarrow \infty} \frac{1}{K^{2(1-\gamma)}} \ln \mathbf{P}\left(\tau \leqslant T_{0}\right) \geqslant-\inf _{v: v+1 \leqslant 0} I(v)=-\frac{1}{2 \mathbf{E} M_{T}^{2}} .
$$

Проверка справедливости верхней оценки

$$
\varlimsup_{K \rightarrow \infty} \frac{1}{K^{2(1-\gamma)}} \ln \mathbf{P}\left(\tau \leqslant T_{0}\right) \leqslant-\frac{1}{2 \mathbf{E} M_{T}^{2}}
$$

намного сложнее. Выберем множество

$$
\mathbf{D}=\left\{u \in \mathbf{C}_{[0, T]}: u_{0}=1 ; u_{t}=u_{\theta(u) \wedge t}, \text { где } \theta(u)=\inf \left\{t: u_{t}=0\right\} \leqslant T\right\}
$$

и заметим, что оно замкнуто в равномерной метрике $(\varrho)$ пространства $\mathbf{C}_{[0, T]}$ непрерывных функций на отрезке $[0, T]$. Поскольку $\left\{\tau_{0} \leqslant T\right\} \subseteq\left\{\left(x_{t}^{K}\right)_{t \in[0, T]} \in \mathbf{D}\right\}$, то естественно вычислить значение

$$
\varlimsup_{K \rightarrow \infty} \frac{1}{K^{2(1-\gamma)}} \ln \mathbf{P}\left(\left(x_{t}^{K}\right)_{t \in[0, T]} \in \mathbf{D}\right) .
$$

Для этого применим ПБУ для семейства $\left\{\left(x_{t}^{K}\right)_{t \in[0, T]}\right\}_{K \rightarrow \infty}$ с параметром $1 / K^{2(1-\gamma)}(!)$ и функционалом действия:

$$
\begin{aligned}
& J_{T}(u)=\frac{1}{2 \sigma^{2}} \int_{0}^{\theta(u) \wedge T}\left(\frac{\dot{u}_{t}-\mu u_{t}}{u_{t}^{\gamma}}\right)^{2} d t, \\
& \text { если } u_{0}=1, \quad d u_{t}=\dot{u}_{t} d t, \quad \int_{[0, \theta(u) \wedge T]}\left[\frac{\dot{u}_{t}-\mu u_{t}}{u^{\gamma}}\right]^{2} d t<\infty,
\end{aligned}
$$

$J_{T}(u)=\infty$ в противном случае

(см. теорему 4.1). В соответствии с теорией больших уклонений,

$$
\varlimsup_{K \rightarrow \infty} \frac{1}{K^{2(1-\gamma)}} \ln \mathbf{P}\left(\left(x_{t}^{K}\right)_{t \in[0, T]} \in \mathbf{D}\right) \leqslant-\inf _{u \in \mathbf{D}} J_{T}(u),
$$

так что осталось показать, что

$$
\inf _{u \in \mathbf{D}} J_{T}(u)=\frac{1}{2 \mathbf{E} M_{T}^{2}} .
$$

Процедура минимизации $J_{T}(u)$ по $u \in \mathbf{D}$ автоматически исключает из рассмотрения функции $u_{t}$ такие, что $d u_{t} \nless d t$ и $\int_{0}^{\theta(u) \wedge T}\left(u_{t}^{\gamma}\right)^{-1}\left(\dot{u}_{t}-\mu u_{t}\right) d t=\infty$. Такая процедура минимизации может быть реализована с помощью некоторой детерминированной задачи оптимального управления с управлением $w_{t}$ и управляемым процессом $u_{t}$, являющимся решением дифференциального уравнения

$$
\dot{u}_{t}=\mu u_{t}+\sigma u_{t}^{\gamma} w_{t}, \quad t \leqslant \theta(u) \wedge T,
$$


с начальным условием $u_{0}=1$. Функция $u_{t}$ принадлежит множеству D. Пара $\left(w_{t}^{*}, \theta\left(u^{*}\right)\right)$ с $u^{*}(t)$, соответствующим $w_{t}^{*}$, оптимальна, если

$$
\int_{0}^{\theta\left(u^{*}\right) \wedge T}\left(w_{t}^{*}\right)^{2} d t \leqslant \int_{0}^{\theta(u) \wedge T} w_{t}^{2} d t
$$

для любой другой пары $\left(w_{t}, u_{t}\right)$ с $\int_{0}^{\theta(u) \wedge T} w_{t}^{2} d t<\infty$. Технически удобно воспользоваться заменой переменных $v_{t}=u_{t}^{1-\gamma}$, которая позволяет использовать линейное дифференциальное уравнение

$$
\dot{v}_{t}=\mu(1-\gamma) v_{t}+\sigma(1-\gamma) w_{t}
$$

с начальным условием $v_{0}=1$ вместо нелинейного уравнения (2.10). Мы также будем использовать тот факт, что $v_{\theta(u)}=0$, если $u_{\theta(u)}=0$, и $v_{\theta(u)}>0$, если $u_{\theta(u)}>0$. Явное решение уравнения $(2.11)$ с условием $\theta(u) \leqslant T$ влечет за собой равенство

$$
0=v_{\theta(u)} e^{-\mu(1-\gamma) t}=\left[1+\sigma(1-\gamma) \int_{[0, \theta(u) \wedge T)} e^{-\mu(1-\gamma) s} w_{s} d s\right],
$$

эквивалентное равенству

$$
-\frac{1}{\sigma(1-\gamma)}=\int_{[0, \theta(u) \wedge T)} e^{-\mu(1-\gamma) s} w_{s} d s,
$$

откуда с помощью неравенства Коши-Шварца-Буняковского получаем

$$
\int_{[0, \theta(u) \wedge T)} w_{t}^{2} d t \geqslant \frac{2 \mu}{\sigma^{2}(1-\gamma)\left[1-e^{-2 \mu(1-\gamma) \theta(u)}\right]} \geqslant \frac{2 \mu}{\sigma^{2}(1-\gamma)\left[1-e^{-2 \mu(1-\gamma) T}\right]} .
$$

Выбор $w_{t}^{*}$ обусловлен двумя требованиями:

1) равенство (2.12) остается справедливым при замене $w_{t}$ на $w_{t}^{*}$;

2) $\int_{\left[0, \theta\left(u^{*}\right) \wedge T\right)}\left(w_{t}^{*}\right)^{2} d t=2 \mu /\left(\sigma^{2}(1-\gamma)\left[1-e^{-2 \mu(1-\gamma) T}\right]\right)$.

Эти требования выполнены для

$$
w_{t}^{*}=-\frac{1}{\sigma} \frac{2 \mu}{1-e^{-2 \mu(1-\gamma) T}} e^{-\mu(1-\gamma) t} .
$$

Следовательно,

$$
\frac{1}{2} \int_{0}^{T}\left(w_{t}^{*}\right)^{2} d t=\frac{1}{2\langle M\rangle_{T}} .
$$

3. Наиболее правдоподобная траектория разорения нормированного процесса $x_{t}^{K}$. Поскольку $u_{t}^{*} \equiv\left(v_{t}^{*}\right)^{1 / \gamma}$, где $v_{t}^{*}$ есть решение уравнения

$$
\dot{v}_{t}^{*}=\mu(1-\gamma) v_{t}^{*}+\sigma(1-\gamma) w_{t}^{*}
$$

с $v_{0}^{*}=1$, то

$$
u_{t}^{*}=e^{\mu t}\left[1-\frac{1-2 \mu\langle M\rangle_{t} /\left(\sigma^{2}(1-\gamma)\right)}{1-2 \mu\langle M\rangle_{T} /\left(\sigma^{2}(1-\gamma)\right)}\right]^{1 /(1-\gamma)} \equiv e^{\mu t}\left[1-\frac{e^{-2(1-\gamma) \mu t}}{e^{-2(1-\gamma) \mu T}}\right]^{1 /(1-\gamma)} .
$$

С другой стороны, по теореме 4.1 с $u_{t}^{*}$ имеет место соотношение

$$
\lim _{\delta \rightarrow 0} \lim _{K \rightarrow \infty} \frac{1}{K^{2(1-\gamma)}} \ln \mathbf{P}\left(\sup _{t \in[0, T]}\left|x_{t}^{K}-u_{t}^{*}\right| \leqslant \delta\right)=-J_{T}\left(u^{*}\right) .
$$

В то же время, по теореме 4.1 с $u \in \mathbf{D}$ получаем аналогичное соотношение:

$$
\begin{gathered}
\lim _{\delta \rightarrow 0} \lim _{K \rightarrow \infty} \frac{1}{K^{2(1-\gamma)}} \ln \mathbf{P}\left(\sup _{t \in[0, T]}\left|x_{t}^{K}-u_{t}\right| \leqslant \delta\right) \\
=-J_{T}(u) \leqslant-\inf _{u \in \mathbf{D}} J_{T}(u)=-J_{T}\left(u^{*}\right) .
\end{gathered}
$$

Следовательно, функция $u_{t}^{*}$ может рассматриваться как наиболее вероятная асимптотическая оценка траектории $x_{t}^{K}$, поглощенной на отрезке времени $[0, T]$. 
4. Принцип больших уклонений для семейства $\left\{\left(x_{t}^{K}\right)_{t \in[0, T]}\right\}_{K \rightarrow \infty}$. Семейство процессов находится в рамках постановки Вентцеля-Фрейдлина [5]. В нашей постановке мы принимаем во внимание, что случайный процесс $x_{t}^{K}$ поглощается в марковский момент $\tau_{0}$, т.е. его траектории принадлежат пространству $\mathbf{C}_{[0, T]}^{\text {abc }}\left(\mathbf{R}_{+}\right)$непрерывных неотрицательных функций $u_{t}=u_{t \wedge \theta(u)}$, где $\theta(u)=\inf \left\{t \leqslant T: u_{t}=0\right\}$, из $\mathbf{C}_{[0, T]}(\mathbf{R})$. Подпространство $\mathbf{C}_{[0, T]}^{\mathrm{abc}}\left(\mathbf{R}_{+}\right)$замкнуто в равномерной метрике $\varrho$, и, следовательно, достаточно проверять ПБУ в $\left(\mathbf{C}_{[0, T]}^{\mathrm{abc}}\left(\mathbf{R}_{+}\right), \varrho\right)$. В то же время, использование $\left(\mathbf{C}_{[0, T]}^{\mathrm{abc}}\left(\mathbf{R}_{+}\right), \varrho\right)$ вместо $\mathbf{C}_{[0, T]}(\mathbf{R})$ позволяет применять стандартный подход с несложными дополнительными деталями.

Теорема 4.1. Семейство $\left\{\left(x_{t}^{K}\right)_{t \geqslant 0}\right\}_{K \rightarrow \infty}$ удовлетворяет ПБУ в метрическом пространстве $\left(\mathbf{C}_{[0, T]}^{\mathrm{abc}}\left(\mathbf{R}_{+}\right), \varrho\right)$ с параметром $1 / K^{2(1-\gamma)}$ и функционалом действия

$$
\begin{aligned}
& J_{T}(u)=\frac{1}{2 \sigma^{2}} \int_{0}^{\theta(u) \wedge T}\left(\frac{\dot{u}_{t}-\mu u_{t}}{u_{t}^{\gamma}}\right)^{2} d t, \\
& \text { если } u_{0}=1, \quad d u_{t}=\dot{u}_{t} d t, \quad \int_{[0, \theta(u) \wedge T]}\left[\frac{\dot{u}_{t}-\mu u_{t}}{u^{\gamma}}\right]^{2} d t<\infty, \\
& J_{T}(u)=\infty \text { в противном случае. }
\end{aligned}
$$

Д о к а з а т е л ь с т в о. Семейство процессов $\left\{\left(x_{t}^{K}\right)_{t \geqslant 0}\right\}_{K \rightarrow \infty}$ экспоненциально плотно (см., например, [8, теоремы 1.3 и 3.1]), т.е.

$$
\begin{aligned}
& \lim _{C \rightarrow \infty} \varlimsup_{K \rightarrow 0} \frac{1}{K^{2(1-\gamma)}} \ln \mathbf{P}\left(\sup _{t \in[0, T]} x_{t}^{K} \geqslant C\right)=-\infty, \\
& \lim _{\Delta \rightarrow 0} \varlimsup_{K \rightarrow 0} \sup _{\vartheta \leqslant T} \frac{1}{K^{2(1-\gamma)}} \ln \mathbf{P}\left(\sup _{t \in[0, \Delta]}\left|x_{\vartheta+t}^{K}-x_{\vartheta}^{K}\right| \geqslant \eta\right)=-\infty,
\end{aligned}
$$

где $\eta-$ произвольное число и $\vartheta-$ марковский момент. При проверке (4.1) величину $x_{t}^{K}$ можно заменить на $\left(x_{t}^{K}\right)^{1-\gamma}$ и, учитывая $(2.2)$, воспользоваться неравенством $\left(x_{t}^{K}\right)^{1-\gamma} \leqslant e^{(1-\gamma) \mu t}\left[1+\int_{0}^{t} e^{(-1-\gamma) \mu s}(1-\gamma) \sigma K^{-(1-\gamma)} d B_{s}\right]$ для заключительного вычисления. В силу (4.1) условие (4.2) можно заменить легко проверяемым условием (здесь $\left.\mathfrak{A}_{C}=\left\{\sup _{t \leqslant T} x_{t}^{\varepsilon} \leqslant C\right\}\right)$ :

$$
\lim _{\triangle \rightarrow 0} \varlimsup_{K \rightarrow 0} \sup _{\vartheta \leqslant T} \frac{1}{K^{2(1-\gamma)}} \ln \mathbf{P}\left(\sup _{[0, \Delta]}\left|x_{\vartheta+t}^{K}-x_{\vartheta}^{K}\right| \geqslant \eta, \mathfrak{A}_{C}\right)=-\infty \quad \forall C>0 .
$$

Для $\theta(u)>T$ доказательство локального ПБУ

$$
\lim _{\delta \rightarrow 0} \lim _{K \rightarrow \infty} \frac{1}{K^{2(1-\gamma)}} \ln \mathbf{P}\left(\sup _{t \in[0, T]}\left|x_{t}^{K}-u_{t}\right| \leqslant \delta\right)=-J_{T}(u)
$$

не отличается от стандартного и поэтому не приводится. Случай $J_{T}(u)=-\infty$, включающий в себя $u_{0} \neq 1, d u_{t} \nless d t$, также проверяется стандартным способом и здесь не приводится.

Асимптотический анализ для случая $u_{0}=1, d u_{t}=\dot{u}_{t} d t, \int_{0}^{\theta(u) \wedge T}\left(\left(\dot{u}_{s}-\right.\right.$ $\left.\left.\mu u_{s}\right) / u_{s}^{\gamma}\right)^{2} d s<\infty, \theta(u) \leqslant T$, использует следующий результат.

Предложение 4.1 ([4, теорема А.6.3]). Для любой абсолютно непрерывной функиии $u=\left(u_{t}\right)_{t \in[0, T]}$, отображающей $[0, T]$ в $\mathbf{R}$, июбой константы $a \in \mathbf{R}$

$$
\int_{0}^{T} I_{\left\{u_{t}=a, \dot{u}_{t} \neq 0\right\}} d t=0 .
$$

Локальный ПБУ. Верхняя граница. Положим $u_{t}^{n}=1 / n \vee u_{t}$ и заметим, что $\theta\left(u^{n}\right)>T$. Более того, $u_{0}^{n}=1, d u_{t}^{n}=\dot{u}_{t}^{n} d t$ и, в силу предложения $4.1, \dot{u}_{t}^{n}=$ 
$\dot{u}_{t} I_{\left\{u_{t}>\frac{1}{n}\right\}} d s$, а также $\int_{0}^{\theta\left(u^{n}\right) \wedge T}\left(\left(\dot{u}_{s}^{n}-\mu u_{s}^{n}\right) /\left(u_{s}^{n}\right)^{\gamma}\right)^{2} d s<\infty$. Поскольку $\tau^{n}=\inf \left\{t: u_{t} \leqslant\right.$ $1 / n\} \rightarrow \theta(u), n \rightarrow \infty$, то

$$
\begin{aligned}
\varlimsup_{\delta \rightarrow 0} & \varlimsup_{K \rightarrow \infty} \frac{1}{K^{2(1-\gamma)}} \ln \mathbf{P}\left(\sup _{t \in[0, T]}\left|x_{t}^{K}-u_{t}\right| \leqslant \delta\right) \\
& \leqslant \varlimsup_{\delta \rightarrow 0} \varlimsup_{K \rightarrow \infty} \frac{1}{K^{2(1-\gamma)}} \ln \mathbf{P}\left(\sup _{t \in\left[0, \tau^{n} \wedge T\right]}\left|x_{t}^{K}-u_{t}\right| \leqslant \delta\right) \\
& \leqslant-\frac{1}{2 \sigma^{2}} \int_{0}^{\tau^{n} \wedge T}\left(\frac{\dot{u}_{t}^{n}-\mu u_{t}^{n}}{\left(u_{t}^{n}\right)^{\gamma}}\right)^{2} d t=-\frac{1}{2 \sigma^{2}} \int_{0}^{\tau^{n} \wedge T}\left(\frac{\dot{u}_{t}-\mu u_{t}}{u_{t}^{\gamma}}\right)^{2} d t \\
& \underset{n \rightarrow \infty}{\longrightarrow}-\frac{1}{2} \int_{0}^{\theta(u) \wedge T}\left(\frac{\dot{u}_{t}-\mu u_{t}}{u_{t}^{\gamma}}\right)^{2} d t .
\end{aligned}
$$

Локальный ПБУ. Нижняя оценка. При $\phi>\delta>0$ имеем

$$
\begin{aligned}
\left\{\sup _{t \in[0, T]}\left|x_{t}^{K}-u_{t}^{n}\right| \leqslant \delta\right\} & =\left\{\sup _{t \in[0, T]}\left|x_{t}^{K}-u_{t}^{n}\right| \leqslant \delta\right\} \cap\left\{\sup _{t \in[0, T]}\left|u_{t}^{n}-u_{t}\right| \leqslant \phi\right\} \\
& \bigcup\left\{\sup _{t \in[0, T]}\left|x_{t}^{K}-u_{t}^{n}\right| \leqslant \delta\right\} \cap\left\{\sup _{t \in[0, T]}\left|u_{t}^{n}-u_{t}\right|>\phi\right\} \\
& \subseteq\left\{\sup _{t \in[0, T]}\left|x_{t}^{K}-u_{t}\right| \leqslant \phi+\delta\right\} \cap\left\{\sup _{t \in[0, T]}\left|u_{t}^{n}-u_{t}\right|>\phi\right\} \\
& \subseteq\left\{\sup _{t \in[0, T]}\left|x_{t}^{K}-u_{t}\right| \leqslant 2 \phi\right\} \cup\left\{\sup _{t \in[0, T]}\left|u_{t}^{n}-u_{t}\right|>\phi\right\} .
\end{aligned}
$$

Для фиксированного $\phi$ найдется номер $n_{\phi}>1 / \phi$ такой, что для любого $n \geqslant n_{\phi}$ множество $\left\{\sup _{t \in[0, T]}\left|u_{t}^{n}-u_{t}\right|>\phi\right\}$ пусто. Следовательно, для достаточно большого номера $n$

$$
\mathbf{P}\left(\sup _{t \in[0, T]}\left|x_{t}^{K}-u_{t}\right| \leqslant 2 \phi\right) \geqslant \mathbf{P}\left(\sup _{t \in[0, T]}\left|x_{t}^{K}-u_{t}^{n}\right| \leqslant \delta\right) .
$$

Это неравенство и предложение 4.1 обеспечивают нижнюю границу

$$
\begin{aligned}
\varliminf_{K \rightarrow \infty} & \frac{1}{K^{2(1-\gamma)}} \ln \mathbf{P}\left(\sup _{t \in[0, T]}\left|x_{t}^{K}-u_{t}\right| \leqslant 2 \phi\right) \\
& \geqslant \frac{\lim _{\delta \rightarrow 0}}{\lim _{K \rightarrow \infty}} \frac{1}{K^{2(1-\gamma)}} \ln \mathbf{P}\left(\sup _{t \in[0, T]}\left|x_{t}^{K}-u_{t}^{n}\right| \leqslant \delta\right) \\
& \geqslant-\frac{1}{2 \sigma^{2}} \int_{0}^{\tau^{n} \wedge T}\left(\frac{\dot{u}_{s}-\mu u_{s}}{u_{s}^{\gamma}}\right)^{2} d s-\frac{1}{2 \sigma^{2}} \int_{\tau^{n} \wedge T}^{T} \frac{\mu^{2}}{n^{2(1-\gamma)}} \\
& \underset{n \rightarrow \infty}{\longrightarrow}-\frac{1}{2 \sigma^{2}} \int_{0}^{\theta(u) \wedge T}\left(\frac{\dot{u}_{s}-\mu u_{s}}{u_{s}^{\gamma}}\right)^{2} d s .
\end{aligned}
$$

\section{СПИСОК ЛИТЕРАТУРЫ}

1. Cox J. C. The constant elasticity of variance option. Pricing model. - J. Portfolio Management, 1997, v. 23, № 2, p. 15-17.

2. Delbaen F., Shirakawa $H$. A note of option pricing for constant elasticity of variance model. Preprint № 96-03. Zürich: ETH, 1996.

3. Donati-Martin C., Rouault A., Yor M., Zani M. Large deviations for squares of Bessel and Ornstein-Uhlenbeck processes. - Probab. Theory Related Fields, 2004, v. 129, № 2 p. 261-289. 
4. Dupuis P., Ellis R.S. A Weak Convergence Approach to the Theory of Large Deviations. New York: Wiley, 1997, 479 p.

5. Freidlin M. I., Wentzell A.D. Random Perturbations of Dynamical Systems. New York: Springer-Verlag, 1984, $326 \mathrm{p}$.

6. Klebaner F. C. Introduction to Stochastic Calculus with Applications. London: Imperial College Press, 2005, 416 p.

7. Klebaner F. C., Liptser R. Likely path to extinction in simple branching models with large initial population. - J. Appl. Math. Stoch. Anal., 2006, Art. ID 60376, 23 p.

8. Liptser R. Sh., Pukhalskii A.A. Limit theorems on large deviations for semimartingales. - Stochastics Stochastics Rep., 1992, v. 38, № 4, p. 201-249.

9. Lu R., Hsu Y.-H. Valuation of standard options under the constant elasticity of variance model. - Internat. J. Business Econom., 2005, v. 4, № 2, p. 157-165.

10. Rogers L. C. G., Williams D. Diffusions, Markov Processes, and Martingales. V. 2. Itô Calculus. Cambridge: Cambridge Univ. Press, 2000, 480 p.

11. Rouault A. Large deviations and branching processes. - Pliska Stud. Math. Bulgar., 2000, v. 13 , p. $15-38$.

Поступила в редакцию 15.IV.2010

(c) 2010 г.

КУЗНЕЦОВА Т. В.*

\section{УТОЧНЕНИЕ ПРЕДЕЛЬНОЙ ТЕОРЕМЫ ДЛЯ МАКСИМУМОВ НЕЗАВИСИМЫХ СЛУЧАЙНЫХ СУММ В СЛУЧАЕ НУЛЕВОЙ АСИММЕТРИИ}

Рассматривается семейство экстремумов вида

$$
Y_{m n}=\max _{1 \leqslant i \leqslant m} \sum_{j=1}^{n} X_{i j}, \quad m, n \geqslant 1,
$$

где $\left\{X_{i j}\right\}, i \geqslant 1, j \geqslant 1$, - независимые одинаково распределенные случайные величины. Исследуется асимптотика $Y_{m n}$ при $m, n \rightarrow \infty$. Проводится уточнение предельной теоремы для случая, когда случайные величины $\left\{X_{i j}\right\}, i \geqslant 1, j \geqslant 1$, имеют нулевую асимметрию и, возможно, отличный от нуля эксцесс.

Ключевые слова и фразы: максимумы, случайные суммы, линейная нормировка, семиинвариант распределения порядка $k$, коэффициент эксцесса, коэффициент асимметрии.

1. Введение. В теории вероятностей и математической статистике широко используются следующие числовые характеристики распределений: коэффициент асимметрии (skewness) $S=\mu_{3} / \sigma^{3}$ и коэффициент эксцесса (kurtosis) $K=\mu_{4} / \sigma^{4}-3$, где $\mu_{k}=(\mathbf{E}(X-\mathbf{E} X))^{k}, \sigma^{2}=\mathbf{D} X$.

Для нормального распределения $S=0$ и $K=0$. Однако, как отмечено в [6, гл. 6], для реальных финансовых данных характерны большие положительные значения эксцесса (порядка единиц или десятков). Поэтому мы исследуем распределения, для которых $S=0$ и $K \neq 0$.

* Московский государственный университет им. М.В.Ломоносова, механикоматематический факультет, Ленинские горы, 119991 Москва, ГСП-1, Россия; e-mail: stv.msu@gmail.com 